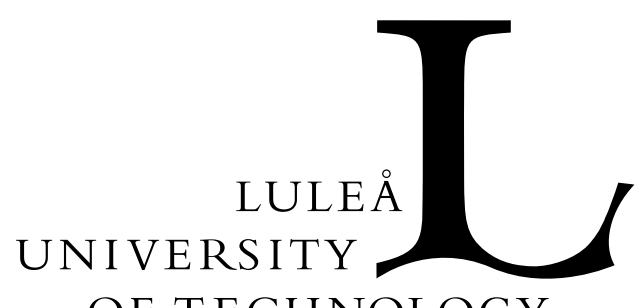

OF TECHNOLOGY

Division of Signal Processing

Visiting address: Universitetsområdet, Porsön, Luleå Postal address: SE-971 87, Luleå, Sweden

Telephone: +46920910 00. Fax: +4692072043

URL: http://www.sm.luth.se/csee/sp/

\title{
Experimental Determination of the Single Point Echo of an Ultrasonic Transducer Using a Tomographic Approach
}

Anders Grennberg and Magnus Sandell

In Proceedings of International Conference of the IEEE Engineering in Medicine and Biology Society (EMBS 92), pp. 2151-2152, Paris, France, October 1992.

(C) 1992 IEEE. Personal use of this material is permitted. However, permission to reprint/republish this material for advertising or promotional purposes or for creating new collective works for resale or redistribution to servers or lists, or to reuse any copyrighted component of this work in other works must be obtained from the IEEE. 


\title{
Experimental determination of the single point echo of an ultrasonic transducer using a tomographic approach
}

\author{
Anders Grennberg Magnus Sandell \\ Division of Signal Processing \\ University of Luleå \\ S-951 87 Luleå, SWEDEN
}

\begin{abstract}
The impulse response method is often used to study the ultrasonic field radiated by planar or gently curved transducers. This, however, requires one transmitting and one receiving transducer which has to be placed with great accuracy in the ultrasonic field. Here we propose a measuring method with only one transducer which acts both as transmitter and receiver. Echoes from sliding halfplanes are registered and by using tomographic methods, the echo from a single point in the same plane as the halfplanes can then be calculated. Beside the ultrasonic field, these calculations will yield us the transducer's characteristics. These can be used to extract further information from an echo of an arbitrary surface, such as the gradient of the surface.
\end{abstract}

\section{INTRODUCTION}

A very common way of imaging with ultrasonics is to use the pulse-echo method [6]. However, there are more information in the echoes besides the arrival time, such as the gradient of the surface at the measured point. To be able to extract this information, a detailed knowledge of the transducer is required, such as the single point echo. In our experiments we have used a focused airborne ultrasonic transducer with a radius of curvature of $45 \mathrm{~mm}$. Two echoes from a plane surface with a sloping angle of $0^{\circ}$ and $5^{\circ}$ respectively are shown in Fig. 1. The shape of the echo is dependent on the angle of inclination [2]. The focal point has the same distance from the transducer in both measurements.

\section{THEORY}

In our experiments we have assumed an axisymmetric focused transducer. The reason for this is simplicity, but the method can be extended to unsymmetric transducers. Suppose that we have a line which lies in a plane parallel to the focalplane and is located at a height $z$ from the transducer. The line has a perpendicular distance $p$ to

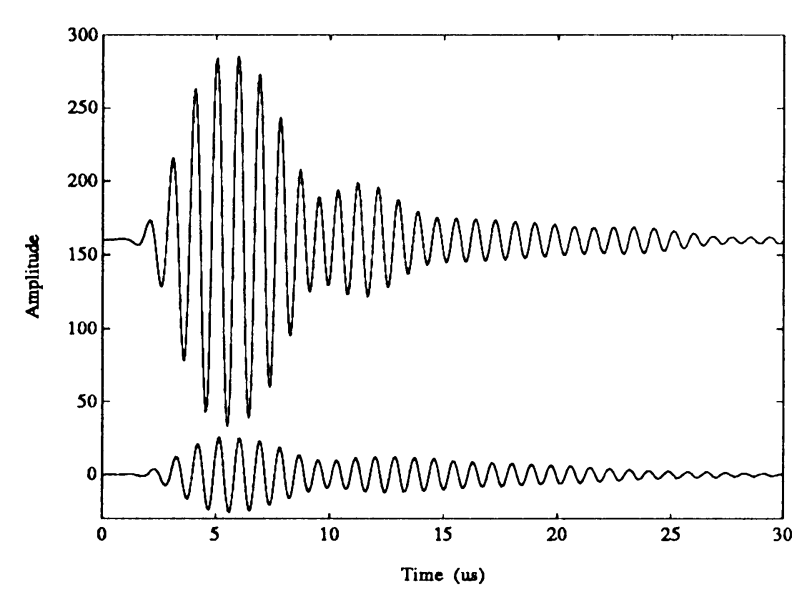

Figure 1: Echoes from plane surfaces. Perpendicular (above) and $5^{\circ}$ angle of inclination (below)

the axis of the transducer, see Fig. 2. The transducer works both as a transmitter and a receiver. The echo $f_{\lambda}(p, z, t)$ from the line will be a function of distance $p$, height $z$ and, of course, time $t$. Since the height $z$ was constant in our experiments and $t$ is only a parameter in our calculations, we will drop them from now on.

If $f(r)$ equals the echo from a single point at a distance $r$ as indicated in Fig. 2, we can write the line-echo, by using superposition, as

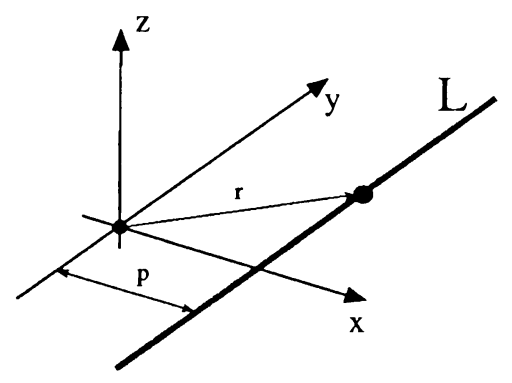

Figure 2: Reflecting line 


$$
\begin{aligned}
& f_{\lambda}(p)=\int_{L} f(r) d r= \\
& 2 \int_{p}^{\infty} f(r) \frac{r}{\sqrt{r^{2}-p^{2}}} d r
\end{aligned}
$$

Since the transducer is axisymmetric, we can assume the line to be parallel to the $y$-axis for the sake of simplicity. The expression in (1) is known as the Abel transform. The inversion of (1) can be found in [1] and is calculated in the following manner:

$$
f(r)=-\frac{1}{\pi} \int_{r}^{\infty} \frac{f_{\lambda}^{\prime}(p)}{\sqrt{p^{2}-r^{2}}} d p
$$

However, there are practical problems in measuring the echo from a line. The line has to be very narrow to be considered a line, which will yield weak echoes with a low SNR. We will also get diffraction effects from the edges if the line isn't infinitesimal thin. These problems can be solved if we instead measure the echoes from halfplanes. If we denote the echo from a perpendicular halfplane with $f_{\pi}(p)$, where $p$ is the same distance as before, we get the following relationship between the line-echo and the halfplane-echo

$$
\begin{gathered}
f_{\pi}(p)=\int_{p}^{\infty} f_{\lambda}(x) d x \\
f_{\lambda}(p, t)=-f_{\pi}^{\prime}(p)
\end{gathered}
$$

Inserting (3) in (2), we obtain the final expression

$$
f(r)=\frac{1}{\pi} \int_{r}^{\infty} \frac{f_{\pi}^{\prime \prime}(p)}{\sqrt{p^{2}-r^{2}}} d p
$$

\section{NUMERICAL EVALUATION}

Inversion algorithms are known for (1), but since we have to differentiate our measurements, we will experience numerical problems. We can also evaluate (4) directly. Since the integrand is singular at its lower bound, this can't be done in a straightforward manner. Our solution to this problem was to use a regularization of the singular integral as shown in [4]. The kernel $1 / \sqrt{p^{2}-r^{2}}$ is replaced with an approximated kernel that has no singularities. We formed the approximated kernel by convolving the original kernel with $\Psi_{\epsilon}(p)$, where $\lim _{\epsilon \rightarrow 0} \Psi_{\epsilon}(p)=\delta(p)$, and $\delta(p)$ is the Dirac function. This will cause the singularity to "smooth" out. Numerous functions $\Psi_{\epsilon}(p)$ are possible to apply and we have tried the most common ones used in tomography.

\section{Results AND Discussion}

The single point echoes are calculated and agrees to the theoretically determined ones. The echo from a point placed at the focal point is shown in Fig. 3. This echo

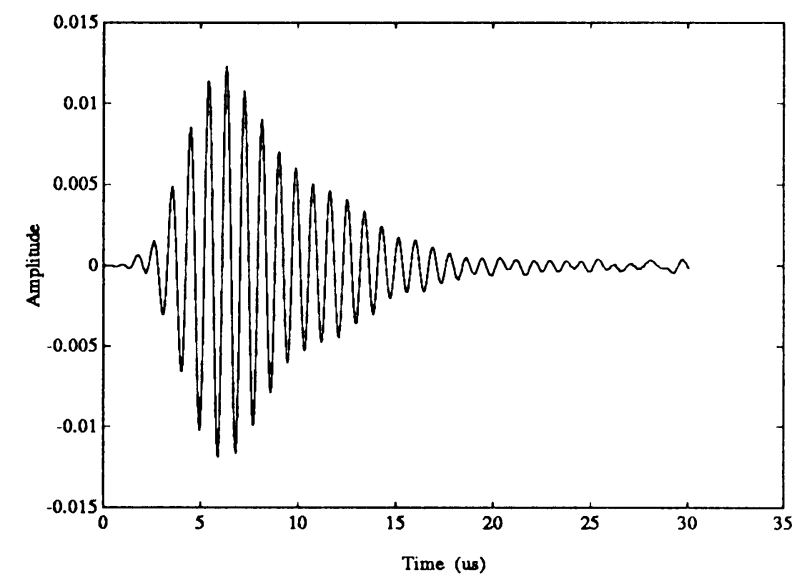

Figure 3: Calculated echo from point reflector at the focal point

is especially interesting, since the transducer's spatial impulse response is equal to a Dirac pulse at the focal point [5]. This means that the echo will be the transducer's acoustoelectric transfer function convolved with itself. The echo from a sloping plane surface is estimated and reasonable agreement is found with the measured echo. The theory can be expanded by using the Radon transform instead of the Abel transform, which allows non-axisymmetrical transducers [3].

\section{References}

[1] Bracewell, R.N.; The Fourier transform and its applications, 2nd ed., McGraw-Hill, 1986.

[2] Börjesson, Per Ola; Holmer, Nils-Gunnar; Lindström, Kjell \& Salomonsson, Göran; "Notes on ultrasound echoes from angular surfaces", Technical report TR-172, Electrical Engineering Department, University of Lund, September 1982.

[3] Deans, Stanley R.; The Radon transform and some of its applications, John Wiley \& Sons, 1983.

[4] Herman, Gabor T. \& Naparstek, Abraham; "Fast image reconstruction based on a Radon inversion formula appropriate for rapidly collected data", Siam J. Appl.Math., vol. 33, no. 3, pp. 511-533, November 1977.

[5] Jensen, J.A.; "A model for the propagation and scattering of ultrasound in tissue", J. Acoust. Soc. Am., vol. 89, no. 1, January 1991.

[6] Sundström, N,; Börjesson, P.O.; Holmer, N.-G.; Olsson, L. \& Persson, H.W.; "Registration of surface structures using airborne focused ultrasound", $U l$ trasound in medicine and biology, vol. 17, no. 5, 1991. 